\title{
Pelvic Rotation and Tilt Can Cause Misinterpretation of the Acetabular Index Measured on Radiographs
}

\author{
M. J. van der Bom PhD, M. E. Groote MD, \\ K. L. Vincken PhD, F. J. Beek PhD, L. W. Bartels PhD
}

Received: 1 June 2010/Accepted: 11 January 2011/Published online: 12 February 2011

(c) The Author(s) 2011. This article is published with open access at Springerlink.com

\begin{abstract}
Background Radiographic diagnosis and followup studies of developmental dysplasia of the hip are commonly performed by measuring the acetabular index on radiographs using Hilgenreiner's method. The outcome of the measurement, however, depends on the orientation of the subject's pelvis relative to the xray source. The influence of pelvic rotation and tilt on the measurement error has been evaluated separately but not in combination.

Questions/purposes We asked whether (1) combinations of pelvic rotation and tilt introduced systematic error in acetabular index measurement in a reproducible way, and (2) ratios proposed to evaluate either pelvic rotation $\left(\mathrm{R}_{\text {rotation }}\right)$ or pelvic tilt $\left(\mathrm{R}_{\text {tilt }}\right)$ are influenced by pelvic tilt and rotation, respectively.

Methods Radiographic measurements of the acetabular index, $\mathrm{R}_{\text {rotation, and }} \mathrm{R}_{\text {tilt }}$ were performed on digitally reconstructed radiographs of one high-resolution
\end{abstract}

Each author certifies that he or she has no commercial associations (eg, consultancies, stock ownership, equity interest, patent/licensing arrangements, etc) that might pose a conflict of interest in connection with the submitted article.

Each author certifies that his or her institution approved the human protocol for this investigation and that all investigations were conducted in conformity with ethical principles of research.

M. J. van der Bom $(\bowtie)$, K. L. Vincken, L. W. Bartels Image Sciences Institute, Department of Radiology, University Medical Center Utrecht, Room Q0S.459, PO Box 85500,

3508 GA, Utrecht, The Netherlands

e-mail: M.vanderBom@umcutrecht.nl

M. E. Groote, F. J. Beek

Department of Pediatric Radiology, University Medical Center Utrecht, Utrecht, The Netherlands three-dimensional CT dataset with various combinations of pelvic rotation and tilt.

Results For rotations and tilt up to $12^{\circ}$, the average systematic errors in the acetabular index varied from $-8.8^{\circ}$ to $4.5^{\circ}$. Negative and positive error values can be interpreted as underestimations and overestimations of the acetabular index, respectively. Errors in acetabular index measurements were acceptable for $\mathrm{R}_{\text {rotation }}$ values between 1.0 and 2.0 and $\mathrm{R}_{\text {tilt }}$ values between 1.1 and 1.8.

Conclusions To limit the systematic error in assessing the acetabular index caused by pelvic misalignment, we recommend only radiographs acquired with $\pm 4^{\circ}$ rotation and $\pm 4^{\circ}$ tilt be considered acceptable.

\section{Introduction}

Developmental dysplasia of the hip (DDH) is a disorder of the acetabulum and proximal femur that can cause a persistent steep acetabulum, possibly leading to an unstable position of the femoral head with subluxation. Early diagnosis and treatment during childhood can restore a normal relationship of acetabulum and femoral head, preventing pain and osteoarthritis in adult years [2].

Although ultrasound has become popular as a diagnostic tool for DDH during the last decades, sensitivity and specificity are yet to be determined [8, 14]. Therefore, radiographic imaging still is used frequently for diagnosis, especially in children with doubtful sonographic findings and in older children to monitor hip development after treatment when ultrasound no longer is possible $[1,6]$.

Radiographic diagnosis of $\mathrm{DDH}$ or followup studies traditionally are performed by measuring the acetabular index (AI), defined as the angle of inclination of the ossified acetabular roof measured on radiographs. A commonly 
used method to measure AI is Hilgenreiner's method [3]. In this method, the AI is defined as the angle between Hilgenreiner's line and the line joining the superolateral margin of the ossified acetabulum to the superolateral margin of the triradiate cartilage. Hilgenreiner's line is a straight line between the $\mathrm{Y}$-shaped triradiate cartilage. If the $\mathrm{AI}$ values exceed 30 for neonates up to 4 months and 25 for children up to 24 months, DDH is suspected $[12,13]$.

The difficulty in consistent AI measurement is precise orientation of the pelvis during radiographic imaging because different orientations of the pelvis will lead to different AI measurements [12]. To obtain a reliable AI measurement using Hilgenreiner's method, the pelvis must be well positioned during acquisition of the radiograph. This is challenging, especially in young children. In the past, the influence of pelvic rotation (rotation of the pelvis around the craniocaudal axis) and of pelvic tilt (rotation of the pelvis around the left-right axis, sometimes also referred to as flexion/extension or inclination) on measurements performed on pelvic radiographs has been investigated [7]. To quantify the quality of the radiograph in terms of pelvic alignment, some parameters have been proposed that are measured on the radiographs. To evaluate pelvic rotation, the ratio between both horizontal diameters of the obturator foramen [12] and the horizontal distance between the symphysis and the sacrococcygeal joint [11] was proposed. For pelvic tilt, these include the angle between the highest medial points of the ischium and the symphysis, the vertical distance between the symphysis and the sacrococcygeal joint, the vertical distance between the upper edge of the symphysis and a line connecting the femoral head centers, the vertical distance between the symphysis and the line connecting the sacroiliac joints, the ratio between the vertical and horizontal diameter of the pelvic foramen, the ratio between the vertical and horizontal points of the obturator foramen, and the ratio between the vertical point of the obturator foramen and the distance between the teardrops [9-13]. From these studies, it was concluded the horizontal and vertical distance between the upper border of the symphysis and the center of the sacrococcygeal joint showed the strongest correlation with pelvic rotation and tilt, respectively. However, the reliability of measuring of these parameters were evaluated on single radiographs obtained from multiple subjects or by varying only the amount of pelvic rotation or pelvic tilt. The effect of pelvic rotation and tilt simultaneously, which is likely to occur in clinical practice, on these parameters is unclear.

We asked whether (1) combinations of pelvic rotation and tilt introduced systematic error in acetabular index measurement in a reproducible way and (2) ratios proposed to evaluate either pelvic rotation or pelvic tilt are influenced by pelvic tilt and rotation, respectively.

\section{Materials and Methods}

Digitally reconstructed radiographs (DRRs) are simulated radiographic images generated by a computer algorithm from a three-dimensional (3-D) CT dataset. Their use is widely accepted in the field of medical image registration $[4,5]$. By using DRRs instead of real radiographs, we were able to vary orientation of the pelvis in a highly controllable fashion by varying the orientation of the 3-D CT dataset while keeping all other imaging parameters fixed. These other parameters include the distance between the center of rotation and the point source $(1145 \mathrm{~mm})$, the distance between the center of rotation and the detector $(100 \mathrm{~mm})$, the detector size $\left(240 \times 180 \mathrm{~mm}^{2}\right)$, and the pixel size $\left(0.1 \times 0.1 \mathrm{~mm}^{2}\right)$, and were taken from the radiographic imaging protocol for radiographic diagnosis of DDH. Our retrospective investigation was approved by the institutional ethical board of University Medical Center Utrecht and informed consent was waived.

We used one high-resolution abdominal CT dataset (in-plane: $0.41 \mathrm{~mm} \times 0.41 \mathrm{~mm}$; through-plane: $0.70 \mathrm{~mm}$ ) of a deceased 3-month-old infant acquired for forensic purposes. The infant had slight hip dysplasia on the left side, which was diagnosed by an experienced pediatric radiologist (FJB) from observation of the CT data in the coronal plane.

DRRs were generated by varying pelvic rotation and tilt. Pelvic rotation was mimicked by rotating the CT volume around the longitudinal axis of the volume. Positive rotation was defined as rotation toward the patient's left acetabulum and negative rotation was defined as rotation toward the patient's right acetabulum. Pelvic tilt was mimicked by rotation of the $\mathrm{CT}$ volume around the axis intersecting the volume from left to right, where positive tilt corresponded to displacement of the symphysis in the caudal direction and negative tilt to displacement of the symphysis in the cephalic direction (Fig. 1). The magnitude of pelvic rotation and tilt was determined by the angular offset in degrees relative to the projection axis (Fig. 1). Pelvic rotation and tilt were varied from $-12^{\circ}$ to $12^{\circ}$ in steps of $4^{\circ}$, leading to a total of 49 DRRs. The center of rotation was placed in the center of the pelvis and the center of the detector was in perfect alignment with the center of rotation and the point source.

Two pediatric radiologists (FJB and MEG) made all 49 measurements of the AI and ratios using in-house developed software (Fig. 2). The parameter evaluating pelvic rotation $\left(\mathrm{R}_{\text {rotation }}\right)$ was defined as the horizontal diameter of the largest obturator foramen divided by the horizontal diameter of the smallest obturator foramen, leading to ratios of 1 and greater. The parameter evaluating pelvic tilt $\left(\mathrm{R}_{\text {tilt }}\right)$ was calculated by the vertical distance between the ossified sacrococcygeal joint and the upper edge of the symphysis, divided by the vertical diameter of 
the obturator foramen, or vice versa. As with the $R_{\text {rotation }}$, the largest vertical distance was divided by the smaller. Average ratios were calculated using measurements of both observers.

During a measurement session, the DRRs were presented in random order. The amount of pelvic tilt and rotation were unknown to the observer. The observer was allowed to enlarge the DRR and to use window leveling and width to improve observation. One of the two experts (FJB) performed a second series of $\mathrm{AI}$ and ratio measurements on all 49 DRRs more than a month after the first for intraobserver analysis. The mean, SD, and 95\% confidence

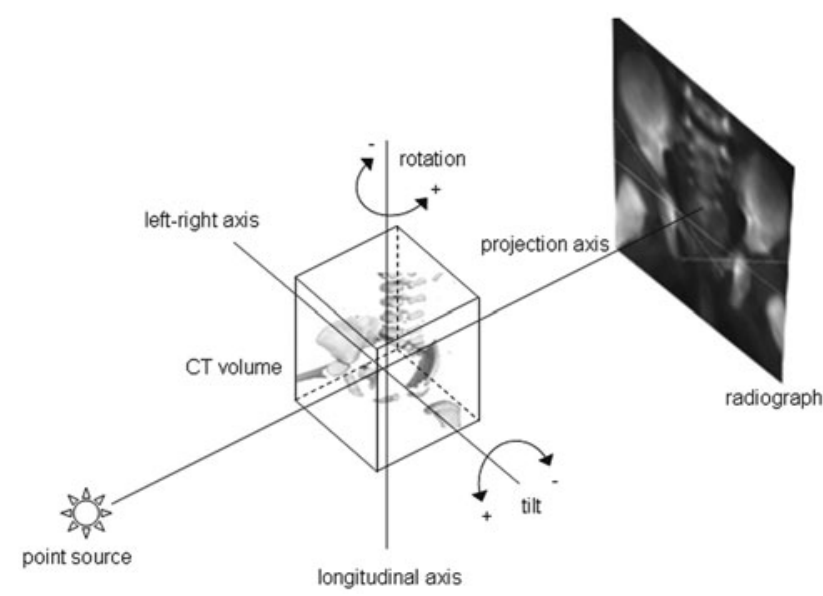

Fig. 1 A schematic representation shows the generation of DRRs and the rotational degrees of freedom of the CT volume. interval of the interobserver and intraobserver differences of the AI measurements were determined using BlandAltman analysis. The mean intraobserver difference was $0.3^{\circ}$ and the SD was $2.1^{\circ}$. The mean interobserver difference for the AI measurements was $0.1^{\circ}$ and the SD was $2.3^{\circ}$. This resulted in a $95 \%$ confidence interval of $4.5^{\circ}$, calculated as $1.96 \times \mathrm{SD}$.

The DRR generated with the pelvis in optimal alignment with the point source and the radiograph was chosen by the observers by visual examination. This ideal pelvic orientation with $0^{\circ}$ rotation and $0^{\circ}$ tilt was defined by an $\mathrm{R}_{\text {rotation }}$ of unity and a position of the sacrococcygeal junction on $2 / 3$ of a line between the symphysis pubis and the caudal extension of the sacrococcygeal joint. The AIs measured with ideal pelvic orientation were used as gold standard indices. Systematic errors in AI measurements caused by nonideal pelvic orientation were determined by the difference between the gold standard index and the measured index. For all orientations, the average systematic error of the two observers was calculated. The average systematic error and the average ratios were evaluated as functions of the pelvic orientation. Some examples of DRRs are shown, including the average AI and ratios measured by the observers (Fig. 3)

\section{Results}

AI measurements were influenced by combinations of pelvic rotation and tilt. The systematic errors induced by
Fig. 2 A screenshot from our inhouse-developed software used for the acetabular angle measurements is shown. The acetabular angles between the dragable lines were calculated automatically and displayed in the user interface.

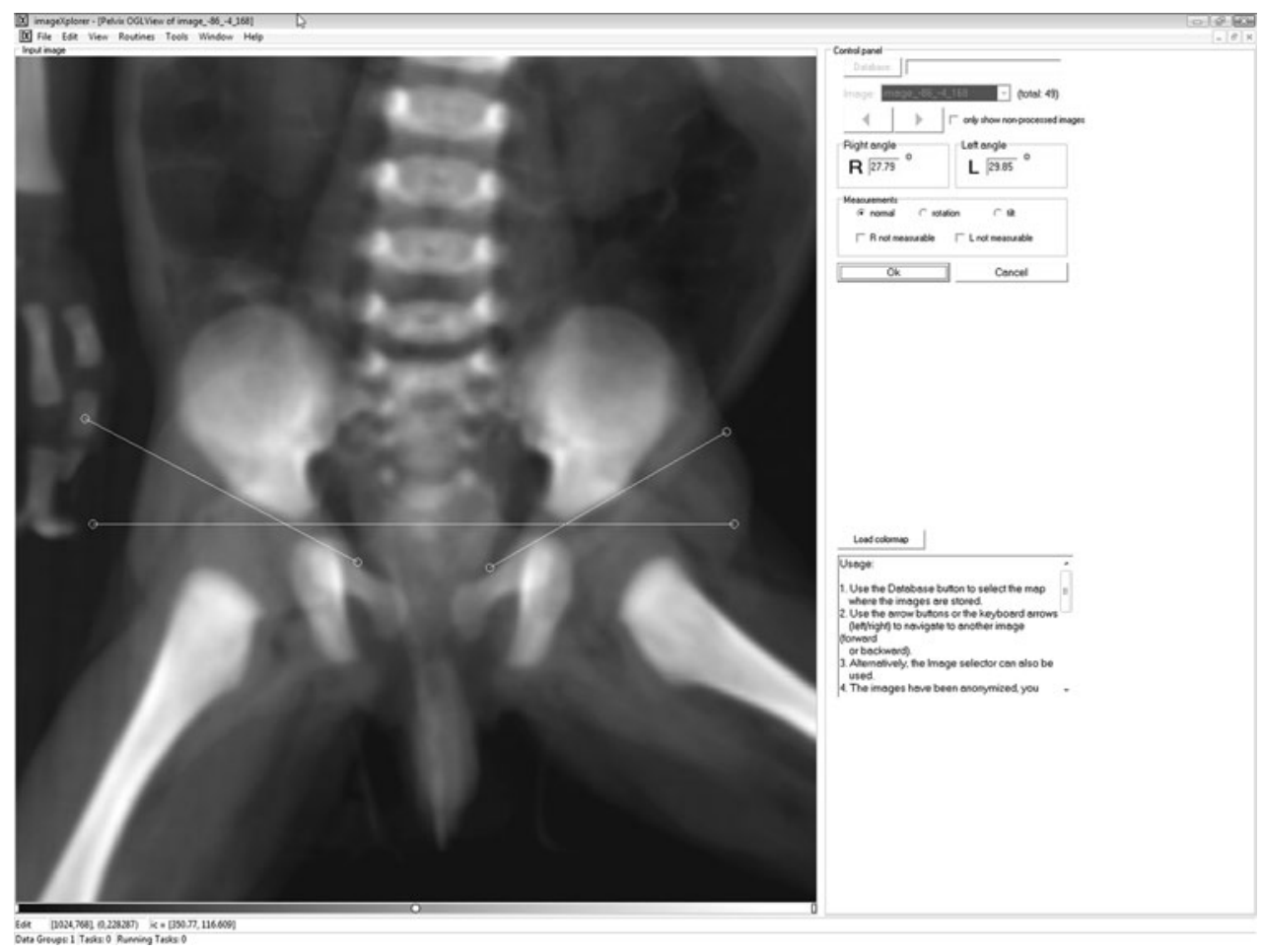




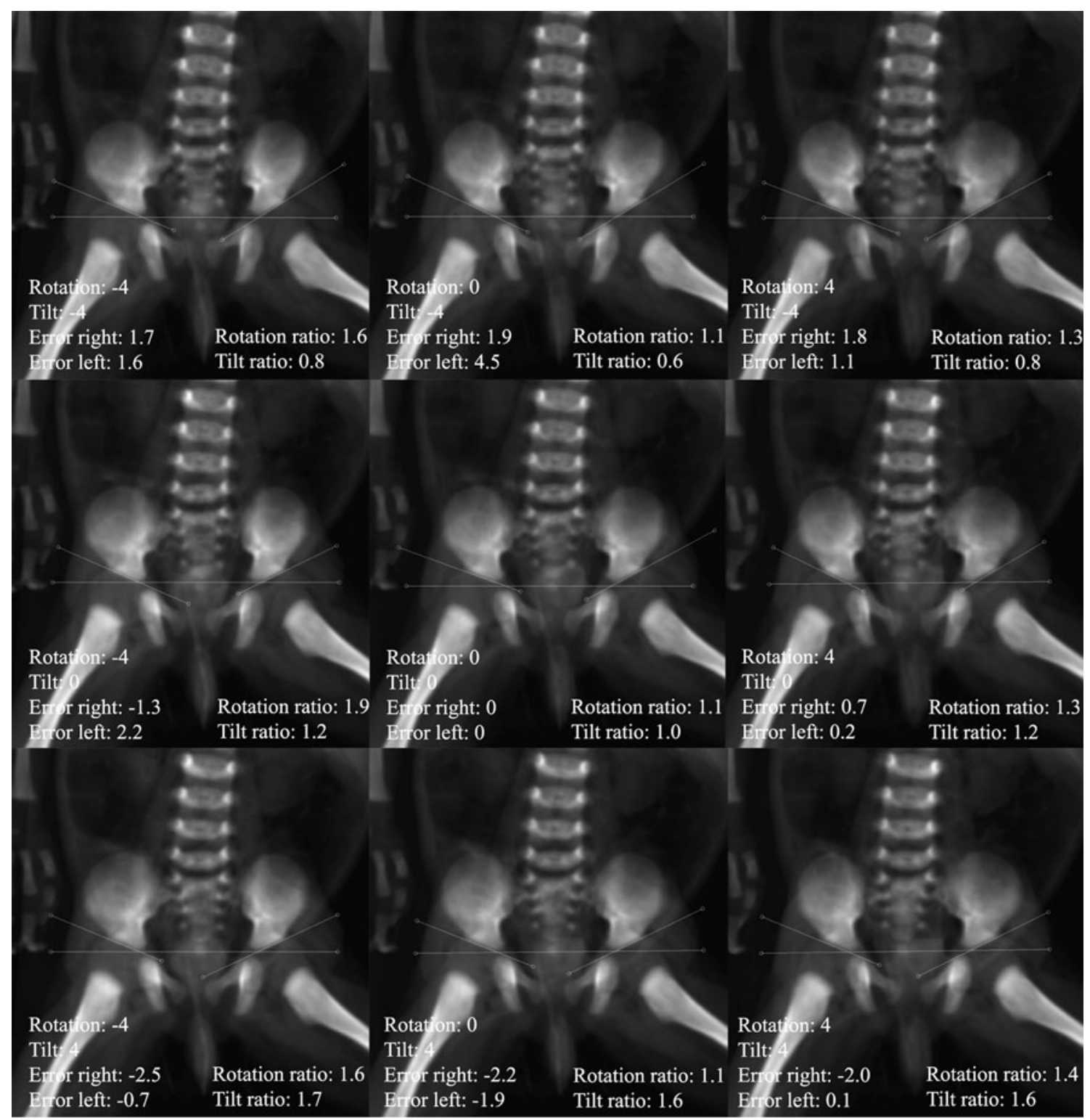

A


B

Fig. 3A-B (A) Some examples of AI measurements performed on DRRs with various pelvic orientations are shown. The center image represents the DRR generated with the ideal pelvic orientation. The DRRs in the top and bottom rows are generated with pelvic $4^{\circ}$ negative and positive tilts, respectively. The DRRs in the left and right columns are generated with $4^{\circ}$ pelvic rotation in the right and left directions, respectively. The average rotation and tilt ratios and the average systematic errors of AI measurements in degrees are indicated in the images. Negative and positive errors correspond to underestimation and overestimations of AI, respectively. (B) Some examples of DRRs generated with $12^{\circ}$ rotation and tilt (left), $0^{\circ}$ rotation and tilt (center), and $-12^{\circ}$ rotation and tilt (right) are shown. 


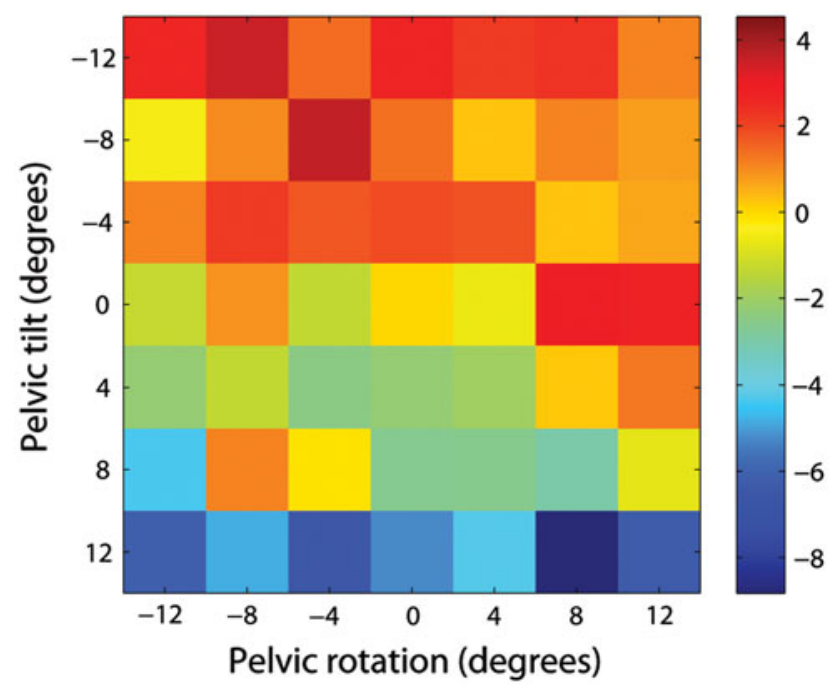

Fig. 4 The average systematic error of the right AI versus pelvic rotation and tilt is shown. Positive numbers along the horizontal axis correspond to pelvic rotation toward the left acetabulum. Positive numbers along the vertical axis correspond to caudal displacement of the symphysis.

rotation and tilt accumulate and can cause either an amplification or compensation of the measurement errors. Underestimation of the AI, corresponding to a negative error, of a pelvis rotated toward the acetabulum under consideration is amplified in the case of an additional positive tilt of the pelvis. The underestimation, however, is reduced when the pelvis also shows negative tilt. In contrast, overestimation of the AI, corresponding to a positive error, of a pelvis rotated toward the opposite direction of the acetabulum under consideration is amplified in the case of an additional positive tilt of the pelvis, whereas an additional negative tilt will reduce the magnitude of overestimation. The average systematic errors in AI measurements introduced by combinations of pelvic rotation and tilt ranged from $-8.8^{\circ}$ to $3.6^{\circ}$ for the right nondysplastic acetabulum (Fig. 4) and from $-8.6^{\circ}$ to $4.5^{\circ}$ for the left acetabulum, which was diagnosed with slight dysplasia (Fig. 5). In these figures, the average systematic errors as a function of pelvic rotation and pelvic tilt are given in a color matrix. Each cell within these matrices represents a combination of pelvic rotation, along the horizontal axis, and tilt, along the vertical axis. The average systematic error corresponding to a combination of rotation and tilt is indicated by a color, of which the magnitude can be derived from the color bar next to the figure. For example, for the right hip, a combination of $0^{\circ}$ rotation and $0^{\circ}$ tilt resulted in an average error of $0^{\circ}$ (orange), and $8^{\circ}$ rotation and $12^{\circ}$ tilt gave an error greater than $8^{\circ}$ (dark blue). The average systematic error in the AI measurements performed on the right acetabulum with pelvic rotations ranging from $-12^{\circ}$ to $12^{\circ}$ and no tilt varied from $-1.3^{\circ}$ to

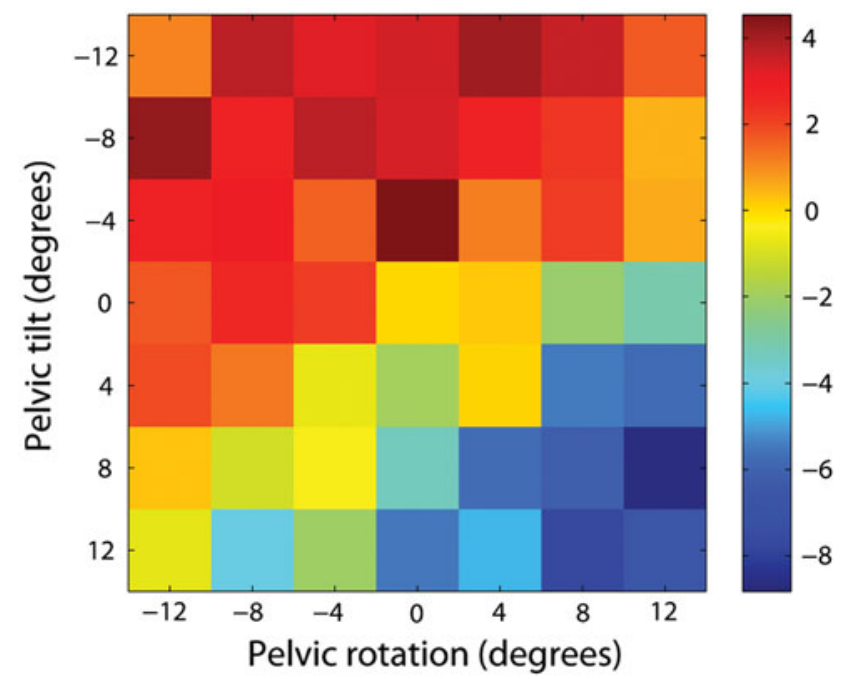

Fig. 5 The average systematic error of the left AI versus pelvic rotation and tilt is shown. Positive numbers along the horizontal axis correspond to pelvic rotation toward the left acetabulum. Positive numbers along the vertical axis correspond to caudal displacement of the symphysis.

$2.9^{\circ}$. Errors in AI measurements caused solely by pelvic tilt ranged from $-5.3^{\circ}$ to $2.7^{\circ}$. For the left acetabulum, the error caused solely by pelvic rotation and by pelvic tilt varied from $-3.1^{\circ}$ to $2.6^{\circ}$ and from $-5.5^{\circ}$ to $4.5^{\circ}$, respectively.

The average rotation ratio $\left(\bar{R}_{\text {rotation }}\right)$ and average tilt ratio $\left(\bar{R}_{\text {tilt }}\right)$ were influenced by changes in pelvic tilt and rotation, respectively. Varying pelvic tilt from $-12^{\circ}$ to $12^{\circ}$ and keeping pelvic rotation fixed at $0^{\circ}$ resulted in $\bar{R}_{\text {rotation }}$ ranging from 1.1 to 1.6 (Fig. 6) and even larger deviations of $\bar{R}_{\text {rotation }}$ were observed for nonzero pelvic rotations. The variance of $\bar{R}_{\text {rotation }}$ measured for $0^{\circ}$ rotation and variable pelvic tilt most likely is caused by small asymmetries between the two obturator foramen. Similarly, $\bar{R}_{\text {tilt }}$ ranged from 1.1 to 1.17 for a fixed pelvic tilt of $0^{\circ}$ and pelvic rotation varying from $-12^{\circ}$ to $12^{\circ}$ (Fig. 7).

\section{Discussion}

Consistent AI measurements are difficult owing to positioning young infants during radiographic imaging. Studies have shown different pelvic orientations lead to different AI measurements $[7,12]$ and have proposed various parameters to assess the amount of pelvic rotation and tilt [9-11]. However, in these studies, analysis was performed by varying only pelvic rotation or tilt separately and their simultaneous effect was not examined. In this study, we investigated whether (1) systematic errors are induced by combinations of pelvic rotation and pelvic tilt, and (2) $R_{\text {rotation }}$, proposed to evaluate the amount of pelvic 


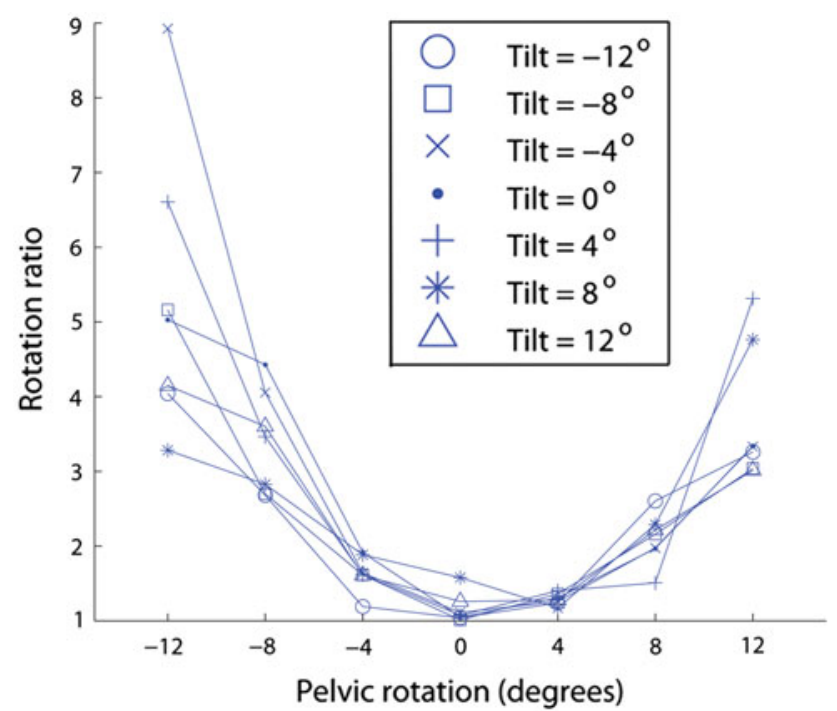

Fig. 6 A graph shows the average rotation ratio as a function of pelvic rotation and constant values of pelvic tilt. Rotation ratio was calculated by the horizontal diameter of the largest obturator foramen divided by the horizontal diameter of the smallest obturator foramen.

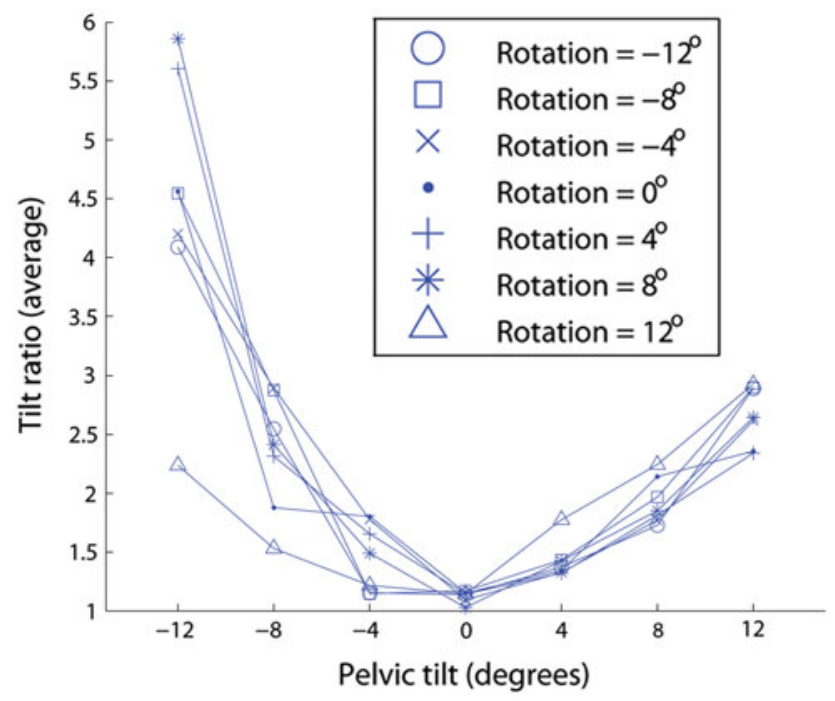

Fig. 7 A graph shows the average tilt ratio as a function of pelvic tilt and constant values of pelvic rotation. Tilt ratio was calculated by dividing the vertical distance between the ossified sacrococcygeal joint and the upper edge of the symphysis by the vertical diameter of the obturator foramen, or vice versa.

rotation, is influenced by pelvic tilt and $\mathrm{R}_{\text {tilt }}$, proposed to evaluate the amount of pelvic tilt, is influenced by rotation of the pelvis.

There are some limitations of our study. As we analyzed the systematic error in AI measurements caused by pelvic rotation and tilt using one subject, the influence of anatomic variations was not included. The goal of our research, however, was to investigate whether combinations of pelvic rotation and tilt induce systematic errors and not to provide absolute error values that will hold in general. By using DRRs generated from a high-resolution CT dataset, we were able to study the influence of rotation and tilt on AI, $R_{\text {rotation }}$, and $R_{\text {tilt }}$ in a highly reproducible fashion. Another limitation is that the accuracies of the radiographic measurements are subject to the degree of ossification of the patient's pelvis. The pelvis of the subject used for our study was not completely ossified. However, this has not endangered our findings, as ossification was sufficient to assess the anatomies of interest.

The literature comprises various studies analyzing the effect of pelvic rotation and tilt on AI measurements. Tönnis [12] observed, for rotation, the AI decreases on the side toward which the rotation takes place, and the $\mathrm{AI}$ on the opposite side increases. In addition, he observed a decreased AI when inclination was increased and an increased AI when inclination was decreased. Portinaro et al. [7] investigated the influence of pelvic rotation and tilt on AI measurements by rotating an xray beam around a postmortem pelvis. They found AI increased when the xray beam was rotated toward the acetabulum that was assessed (simulating rotation of the pelvis toward the opposite acetabulum) and decreased on the opposite side. Cephalic rotation of the xray beam (simulating an extension of the pelvis, corresponding to positive tilt in our study) caused an increment of the AI, and caudal rotation of the xray beam (simulating flexion of the pelvis, corresponding to negative tilt in our study) caused a decrement of the AI. Findings with respect to pelvic rotation were confirmed in our study. Our observations with respect to pelvic tilt were in agreement with those of Tönnis [12], but opposite those reported by Portinaro et al. [7]. In our study, positive tilt (which corresponds to cephalic rotation of the xray source) led to decreased AI and negative tilt (corresponding to caudal rotation of the xray source) increased AI. Furthermore, Portinaro et al. [7] concluded errors in the AI measurements caused by pelvic rotation within $\pm 5^{\circ}$ and pelvic tilt within $\pm 10^{\circ}$ were only $1^{\circ}$ and therefore could be ignored. However, in their study, the influence of only one parameter (either rotation or tilt) at a time was considered. In reality, it is more likely pelvic misalignment during acquisition of the radiograph is caused by a combination of rotation and tilt. In our study, a combination of $4^{\circ}$ rotation and $8^{\circ}$ tilt led to underestimation of the $\mathrm{AI}$ of as much as $8.7^{\circ}$.

To quantify the quality of the radiograph, various parameters were proposed that evaluate pelvic rotation or pelvic tilt [9-13]. Portinaro et al. [7] studied the obturator foramen ratio (corresponding to $\mathrm{R}_{\text {rotation }}$ ) as a function of pelvic rotation. They showed the ratio increases as pelvic rotation is increased and ratios between 0.5 and 2 correspond to pelvic rotations within a range of $\pm 5^{\circ}$. Tannast et al. [10] analyzed various parameters estimating pelvic tilt as a function of pelvic tilt only and concluded the 
vertical distance between the upper edge of the symphysis and the midpoint of the sacrococcygeal joint [9] showed the strongest correlation with pelvic tilt. We showed $\mathrm{R}_{\text {rotation }}$ was not only dependent on rotation but also was influenced by pelvic tilt. Similarly, $\mathrm{R}_{\text {tilt }}$ was not only affected by pelvic tilt but also by the amount of rotation induced. These effects should be taken into consideration when the quality of a radiograph is evaluated using these and similar parameters.

Based on our results, we believe pelvic rotation and tilt should be considered simultaneously during diagnosis of DDH using Hilgenreiner's method. Changing pelvic orientation by inducing rotation and tilt simultaneously can cause systematic errors in the AI that are larger than when only rotation or tilt is considered. To obtain consistent AI measurements, we advise limiting the systematic errors caused by pelvic misalignment to the size of the $95 \%$ confidence interval of the interobserver error. In our study, this results in a maximal acceptable systematic error of $4.5^{\circ}$. This means radiographs acquired with approximately $\pm 4^{\circ}$ rotation and $\pm 4^{\circ}$ tilt should be considered acceptable, which corresponds to $\mathrm{R}_{\text {rotation }}$ values between 1.0 and 2.0 and $\mathrm{R}_{\text {tilt }}$ values between 1.1 and 1.8.

Acknowledgment We thank Dr. R. A. J. Nievelstein for time and effort in improving this research.

Open Access This article is distributed under the terms of the Creative Commons Attribution Noncommercial License which permits any noncommercial use, distribution, and reproduction in any medium, provided the original author(s) and source are credited.

\section{References}

1. Dezateux C, Rosendahl K. Developmental dysplasia of the hip. Lancet. 2007;369:1541-1552.
2. Furnes O, Lie SA, Espehaug B, Vollset SE, Engesaeter LB, Havelin LI. Hip disease and the prognosis of total hip replacements: a review of 53,698 primary total hip replacements reported to the Norwegian Arthroplasty Register 1987-99. J Bone Joint Surg Br. 2001;83:579-586.

3. Hilgenreiner $H$. Zur Frühdiagnose und Frühbehandlung der angeboren Hüftgelenks-verrenkung. Med Klin. 1925;21:13851388.

4. Lemieux L, Jagoe R, Fish DR, Kitchen ND, Thomas DG. A patient-to-computed-tomography image registration method based on digitally reconstructed radiographs. Med Phys. 1994;21: 1749-1760.

5. Markelj P, Tomaževič D, Likar B, Pernuš F. A review of 3D/2D registration methods for image-guided interventions. Med Image Anal. 2010 April 13. [Epub ahead of print].

6. Pirpiris MM, Payman K, Otsuka NY. The assessment of acetabular index: is there still a place for plain radiography? J. Pediatr Orthop. 2006;26:310-315.

7. Portinaro NM, Murray DW, Bhullar TP, Benson MK. Errors in measurement of acetabular index. J Pediatr Orthop. 1995;15: 780-784.

8. Roposch A, Moreau NM, Uleryk E, Doria AS. Developmental dysplasia of the hip: quality of reporting of diagnostic accuracy for US. Radiology. 2006;241:854-860.

9. Siebenrock KA, Kalbermatten DF, Ganz R. Effect of pelvic tilt on acetabular retroversion: a study of pelves from cadavers. Clin Orthop Relat Res. 2003;407:241-248.

10. Tannast M, Murphy SB, Langlotz F, Anderson SE, Siebenrock KA. Estimation of pelvic tilt on anteroposterior X-rays: a comparison of six parameters. Skeletal Radiol. 2005;35:149-155.

11. Tannast M, Zheng G, Anderegg C, Burckhardt K, Langlotz F, Ganz R, Siebenrock KA. Tilt and rotation correction of acetabular version on pelvic radiographs. Clin Orthop Relat Res. 2005;438:182-190.

12. Tönnis D. Normal values of the hip joint for the evaluation of X-rays in children and adults. Clin Orthop Relat Res. 1976;119: 39-47.

13. Tönnis D, Brunken D. [Differentiation of normal and pathological acetabular roof angle in the diagnosis of hip dysplasia: evaluation of 2294 acetabular roof angles of hip joints in children] [in German]. Arch Orthop Unfallchir. 1968;64:197-228.

14. Woolacott NF, Puhan MA, Steurer J, Kleijnen J. Ultrasonography in screening for developmental dysplasia of the hip in newborns: systematic review. BMJ. 2005;330:1413-1418. 